lowing chapter discussing broadly reactive T-cell epitopes and their implications for vaccine design.

The book then moves to a discussion of general principles of vaccinology, beginning with a well-written overview of modern adjuvants. This section is organized primarily by methods of antigen delivery and includes the development of peptide-based vaccines and the use of biodegradable polymers for protein antigen delivery. Recent developments in genetic approaches for vaccine delivery are introduced, including the use of recombinant bacteria, recombinant viruses and naked DNA for antigen delivery. While individual chapters are well done, the reader is left hanging without a unifying discussion of potential mechanisms of immunization or rationale for the pursuit of one recombinant strategy versus another. Also noticeably absent from this section is any discussion of the potential use of dendritic cells as vehicles for antigen delivery, either through adoptive transfer or by in vivo targeting.

The book concludes with a series of reviews on vaccination strategies against

\section{Between Bench and Bedside:}

\section{SCIENCE, HEALING AND INTERLEUKIN-2 IN A CANCER WARD \\ by Ilana Lowy \\ Harvard University Press \\ 1997 ISBN: 0-674-06809-2 \$39.95}

\section{REVIEWED By RALPH A. REISFELD}

Head, Division of Tumor Cell Biology

Department of Immunology, IMM-13

The Scripps Research Institute

10550 North Torrey Pines Road

La Jolla, California 92037, USA

Between Bench and Bedside provides the reader with a remarkable view of the culture of clinical experimentation in oncology, in particular, a view of the interactions between the biology laboratory and the oncology ward. In this book, a clinical trial of interleukin-2 (IL-2) begun in 1986 at the Cancer Foundation, a major French cancer treatment center, is critically examined in an attempt to answer the question "What can be learned from the study of a semiroutine clinical experiment in a cancer treatment center?" The author, an immunologist and science historian, observed the several small clinical trials comprising the Cancer Foundation's IL-2 trial from its be- specific pathogens, covering AIDS, malaria, schistosomiasis and Helicobacter pylori - an effective method to tie together general principles introduced in previous chapters. The topics are generally well selected, giving a representative overview of current challenges in vaccine design. A chapter addressing cancer vaccines, discussing both the similarities with immunization strategies aimed at infectious diseases and some of the unique challenges of antitumor immunization, would have strengthened this section. The closing chapter on vaccination therapy against autoimmune disease lucidly demonstrates the potentially broad applicability of modern vaccinology.

Overall, this book is a remarkable accomplishment. The reader is left with a clear impression that modern vaccinology is firmly founded in basic science advances from a number of disciplines, and that modern vaccine design is truly "rational" vaccine design. I would recommend this book to anyone with an interest in vaccine development. Those in this field who desire a broader perspective should find this book particularly useful.

ginning until July 1990. By using perspectives borrowed from the histories of science and medicine, the sociology of scientific knowledge, the ethnography of the laboratory and the sociology of medicine, she creates a very realistic picture of this and other clinical trials. She takes a detailed look at the collaborations between scientists and clinicians, two very different groups with a common goal to develop a cure for cancer.

Even though the narrative is occasionally patchy and heterogeneous, she offers a superb analysis in Chapters 1 through 3

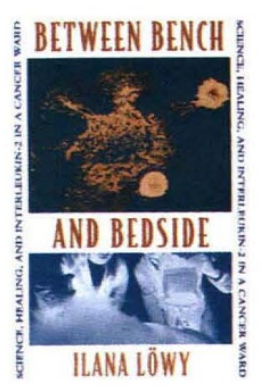

anomas. The overall response rate of IL-2 in the Cancer Foundation's trials was $20-25 \%$, which was similar to that reported in other trials in Israel and the United States. Even though these results were not as good as initially hoped, they nevertheless received favorable press coverage that lead, in 1993, to the creation of a clinical biology division that provided the nucleus for a new laboratory complex. It was hoped that this complex would eventually gather together analysis and clinical research laboratories, as well as laboratories dedicated to preclinical and industrial research.

In Chapter 5, the author describes in considerable detail the professional cultures, jurisdictions and practices that were involved in making the IL-2 trial work. She recounts how "material, social and discursive techniques, shared objects and concepts, control over resources and persons and the development of a specific 'intermediary zone' facilitated the development of efficient links" between the immunologists, oncologists and industrial IL-2 producers participating in this effort.

Finally in Chapter 6 the author summarizes the results of the trial and reflects on a "science-laden" pathology (that is, the direct dependence of therapeutic interventions in cancer on laboratory findings) between bench and bedside that leads to rich and complex interactions between a new medical practice, such as the introduction of IL-2, to cancer therapy and its "environment," that is, the other practices, claims and organizations that make up the culture of clinical experimentation in oncology. In the end, one can certainly agree of the historical and social background of the Cancer Foundation's IL-2 trial. The development of a distinct culture of clinical experimentation in the oncology clinics is clearly described, as is the application of immunological methods to cancer therapy and the specific story of IL-2 as an anticancer drug. The author's personal view of this clinical trial is both scholarly and realistic.

Administration of IL-2 alone or, in some trials, with either the patient's own lymphokine-activated killer cells or tumor-infiltrating lymphocytes to enhance the antitumor effect resulted in only a few "miracles" - complete and stable regression of disseminated mel- with the author's conclusion that as long as cancer is defined as a disease of "deviant cells" lacking an efficient treatment and as long as cancer therapy is structured as a strongly science-driven area of medical intervention, weakly dependent on life-style considerations, connections between the biology laboratory and the oncology ward will remain stable.

Between Bench and Bedside makes for excellent reading and can be highly recommended to immunologists and those among scientists and the public who seek to know more about the challenges involved in developing effective treatments for human malignancies. 